TITLE:

\title{
Effects of Al doping on the thermoelectric performance of CoSi single crystal
}

$\operatorname{AUTHOR}(S):$

Li, C.C.; Ren, W.L.; Zhang, L.T.; Ito, K; Wu, J.S.

CITATION:

Li, C.C.... [et al]. Effects of Al doping on the thermoelectric performance of CoSi single crystal. JOURNAL OF APPLIED PHYSICS 2005, 98(6): 063706.

ISSUE DATE:

2005-09-15

URL:

http://hdl.handle.net/2433/39716

RIGHT:

Copyright 2005 American Institute of Physics. This article may be downloaded for personal use only. Any other use requires prior permission of the author and the American Institute of Physics. 


\title{
Effects of Al doping on the thermoelectric performance of CoSi single crystal
}

\author{
C. C. Li, W. L. Ren, and L. T. Zhang ${ }^{\text {a) }}$ \\ Key Laboratory of the Ministry of Education for High Temperature Materials and Testing, \\ School of Materials Science and Engineering, Shanghai Jiao Tong University, Shanghai 200030, \\ People's Republic of China \\ K. Ito \\ Department of Materials Science and Engineering, Kyoto University, Sakyo-ku, Kyoto 606-8501, Japan \\ J. S. Wu \\ Key Laboratory of the Ministry of Education for High Temperature Materials and Testing, \\ School of Materials Science and Engineering, Shanghai Jiao Tong University, Shanghai 200030, \\ People's Republic of China
}

(Received 2 March 2005; accepted 1 August 2005; published online 19 September 2005)

\begin{abstract}
Thermoelectric transport properties of $\mathrm{CoSi}_{1-x} \mathrm{Al}_{x}$ single crystals have been measured over the temperature range from 300 to $973 \mathrm{~K}$ to investigate the effects of Al substitution on the electrical resistivity, Seebeck coefficient, and thermal conductivity. The solubility limit of Al substitution for $\mathrm{Si}$ in $\mathrm{CoSi}$ is found to be in the range of $0.2 \leqslant x<0.3$. The electrical resistivity increases with increasing $\mathrm{Al}$ substitution $x$ and reaches a maximum at $x=0.08$. When $x$ is further increased, there is a drop in the electrical reisitivity between 300 and $550 \mathrm{~K}$ and there is almost no change at higher temperatures. The Seebeck coefficients of $\mathrm{CoSi}_{1-x} \mathrm{Al}_{x}$ single crystals shift towards the positive direction compared to that of the binary crystal over the entire measured temperature range. The Seebeck coefficient decreases as a function of temperature. The thermal conductivity and the figure of merit decrease significantly with increasing $\mathrm{Al}$ content. The decrease in thermal conductivity is mainly attributed to the additional scattering from $\mathrm{Al}$ atoms. (c) 2005 American Institute of Physics.
\end{abstract}

[DOI: $10.1063 / 1.2041843$ ]

\section{INTRODUCTION}

Some semiconducting transition-metal silicides have attracted considerable attention due to their practical applications in electronics and thermoelectrics. ${ }^{1} \mathrm{CoSi}$ with a $\mathrm{B2}$ cubic structure has been reported to be a candidate for advanced thermoelectric applications. ${ }^{2-5}$ Band structure calculations ${ }^{1,6,7}$ show that there is a slight overlap of conduction and valence bands at the Femi level, indicating that $\mathrm{CoSi}$ is a semimetal. Previous studies ${ }^{1}$ on this compound indicated a moderate Seebeck coefficient value of $-83 \mu \mathrm{V} / \mathrm{K}$ at room temperature and a carrier concentration of about $10^{20} \mathrm{~cm}^{-3}$. At room temperature, the extra carriers are electrons. The effective masses for holes and electrons are $6 m_{e}$ and $2 m_{e}$, respectively. ${ }^{1,3}$ In order to improve the thermoelectric performance of $\mathrm{CoSi}$, doping has been attempted in this compound. It is known that CoSi forms a continuous solid solution with $\mathrm{FeSi}$ and dissolves up to $10 \% \mathrm{NiSi}$. The electron concentration increases with $\mathrm{Ni}$ substitution, but decreases with $\mathrm{Fe}$ substitution. Doping with $\mathrm{Fe}$ and $\mathrm{Ni}$ on the Co site resulted in increased electrical and thermal conductivities, especially at low temperatures. ${ }^{1,2}$ However, the thermoelectric power is decreased by $\mathrm{Fe}$ and Ni substitutions. ${ }^{1,2}$

In recent years, doping with a third element on the Si site in $\mathrm{CoSi}$ has been attempted. Doping by $\mathrm{B}, \mathrm{Ge}$, and $\mathrm{Al}$ on the $\mathrm{Si}$ site has been reported in several recent works. ${ }^{8-10}$ In a

\footnotetext{
a) Author to whom correspondence should be addressed; FAX: +86-2152540011; electronic mail: lantingzh@sjtu.edu.cn
}

recent paper, ${ }^{10}$ the thermoelectric properties of polycrystalline $\mathrm{Al}$-doped $\mathrm{CoSi}$ were reported in a temperature range of $0-300 \mathrm{~K}$. In the present work, the Al-doped crystals of $\mathrm{CoSi}$ have been prepared by an optical-heating floating zone method. The solubility limit of $\mathrm{Al}$ substitution for $\mathrm{Si}$ has been determined. The effects of Al doping on the thermoelectric transport properties have been investigated in the temperature range of $300-973 \mathrm{~K}$ using single crystals to eliminate the effects from cast defects and grain boundaries. A possible mechanism has been discussed.

\section{EXPERIMENTAL PROCEDURES}

Ingots of $\mathrm{CoSi}_{1-x} \mathrm{Al}_{x}(x=0-0.5)$ were prepared by melting high-purity Co (99.98\%), $\mathrm{Si}$ (99.999\%), and $\mathrm{Al}$ (99.999\%) in a laboratory-scale nonconsumable arc-melting furnace under a purified Ar atmosphere. The ingots were remelted six times and annealed at $1473 \mathrm{~K}$ for $24 \mathrm{~h}$ to ensure chemical homogeneity. Some of the ingots $(x=0.0,0.04$, $0.08,0.12$, and 0.16 ) were melted into rods about $10 \mathrm{~mm}$ in diameter and $80 \mathrm{~mm}$ in length to grow single crystals. Crystal growth was performed in an optical-heating floating zone melting apparatus (Asgal FZ-20035 WHV) at a growth rate of 7-10 mm/hr in a high-purity Ar gas flow. Crystal structure, composition, microstructure, and perfection of the obtained ingots and crystals were characterized by powder x-ray diffraction $(\mathrm{XRD}, \mathrm{Cu} K \alpha)$ and electron probe microanalyzer (EPMA). The orientation of the single crystals was determined by the Laue back-reflection method. 

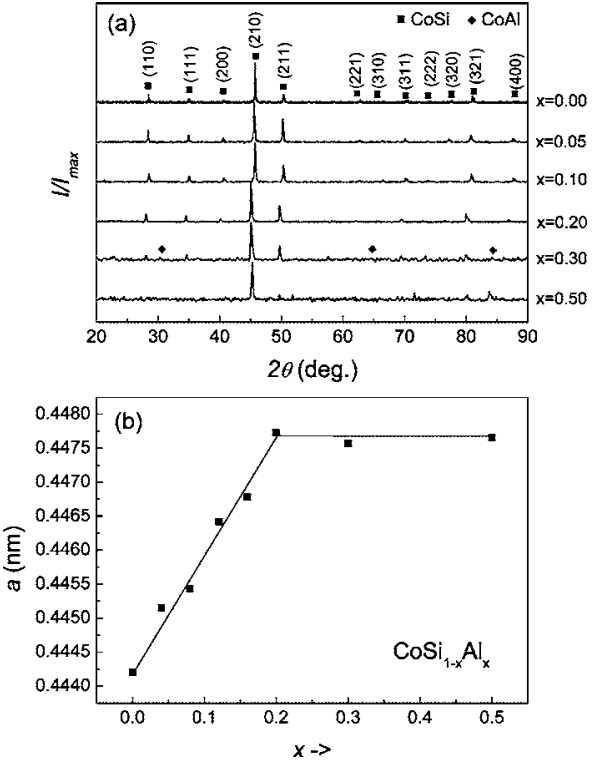

FIG. 1. (a) X-ray-diffraction patterns of $\operatorname{CoSi}_{1-x} \mathrm{Al}_{\mathrm{x}}$ and (b) lattice parameter vs $\mathrm{Al}$ concentration as obtained from x-ray diffraction.

Electrical resistivity $\rho$ and Hall coefficient $R_{H}$ were measured in a temperature range from 300 to $973 \mathrm{~K}$ using the van der Pauw technique under an argon atmosphere. Carrier concentration $n$ was determined from the measured Hall coefficient $R_{H}$ using the $n=1 / R_{H} e$ relation, where $e$ is the electron charge. Seebeck coefficient $S$ was measured by applying a small temperature difference $(3 \mathrm{~K})$ to the two ends of a specimen in a temperature range from 300 to $973 \mathrm{~K}$ under an argon atmosphere. Thermal conductivivty $\kappa\left(\kappa=D C_{p} d\right)$ was calculated from the experimental values of thermal diffusivity $D$, specific heat capacity $C_{p}$, and density $d$. The thermal diffusivity was measured by an ac calorimetry method from 300 to $473 \mathrm{~K}$. The specific heat capacity was measured by a differential scanning calorimeter (DSC) in an Ar flow.

\section{RESULTS AND DISCUSSIONS}

Figures 1(a) and 1(b) show the x-ray-diffraction patterns of $\mathrm{CoSi}_{1-x} \mathrm{Al}_{x}$ and the determined lattice parameter as a function of $\mathrm{Al}$ concentration, respectively. When $x \leqslant 0.2$, the $\mathrm{CoSi}_{1-x} \mathrm{Al}_{x}$ ingots are all of monolithic CoSi phase, whose lattice parameter increases linearly with increasing $x$. This indicates that the Si sites are substituted by $\mathrm{Al}$ atoms according to Vegard's law, which is consistent with a previous work. ${ }^{10}$ When $x \geqslant 0.30, B 2$-type CoAl phase besides CoSi is identified in the microstructure as indicated in Fig. 1(a). The lattice parameter of the CoSi phase is almost constant in this composition range. Figure 2 displays a typical backscattered electron image of $\mathrm{CoSi}_{0.7} \mathrm{Al}_{0.3}$. The analyzed compositions of phases " $\mathrm{A}$ " and "B" are listed in Table I. The Co contents in the two phases are very close to 50 at. \%, while A is obviously richer in $\mathrm{Al}$ than $\mathrm{B}$ is. The determined composition of phase A (B20-type CoSi structure) can be expressed as $\mathrm{Co}_{1.06} \mathrm{Si}_{0.77} \mathrm{Al}_{0.23}$. Therefore, it can be concluded that the solubility limit of $\mathrm{Al}$ in $\mathrm{CoSi}_{1-x} \mathrm{Al}_{x}$ is in the range of 0.2 $\leqslant x<0.3$

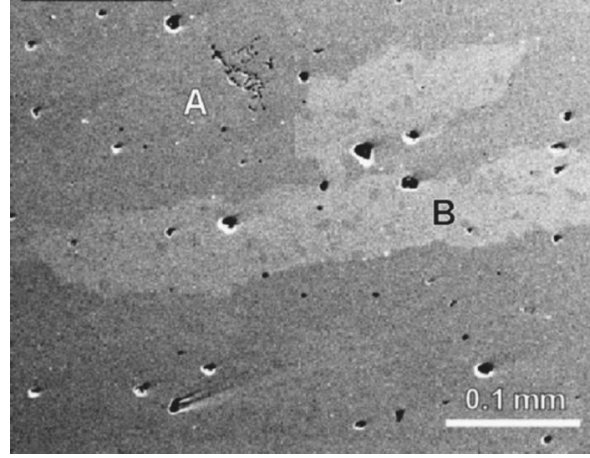

FIG. 2. Backscattered electron image of $\mathrm{CoSi}_{0.7} \mathrm{Al}_{0.3}$ annealed at $1473 \mathrm{~K}$ for 24 h (A: CoSi phase, B: CoAl phase).

The temperature dependence of the electrical resistivity of $\mathrm{CoSi}_{1-x} \mathrm{Al}_{x}$ is shown in Fig. 3(a), compared with the data for binary $\mathrm{CoSi}$ (dashed line) reported in the literature. ${ }^{1} \mathrm{It}$ can be found that the electrical resistivity increases dramatically with increasing $\mathrm{Al}$ concentration in $\mathrm{CoSi}_{1-x} \mathrm{Al}_{x}$ and reaches a maximum at $x=0.08$. Further increase of $\mathrm{Al}$ concentration results in a slight decrease of electrical resistivity in the temperature range from 300 to $550 \mathrm{~K}$; while the electrical resistivity is almost unchanged at higher temperatures [Fig. 3(a)]. Figure 3(b) compares the dependence of roomtemperature electrical resistivity on $\mathrm{Al}$ concentration $x$ in $\mathrm{CoSi}_{1-x} \mathrm{Al}_{x}$. For binary $\mathrm{CoSi}$, the electrical resistivity of $\mathrm{CoSi}$ in this study is slightly higher than that in Ref. 1 and is almost one order of magnitude smaller than that in Ref. 10. In Ref. 10, it shows that the electrical resistivity decreases continuously with increasing $x$, which is fully different from our results. Since the specimens used in Ref. 10 were arcmelted polycrystals, the measured electrical resistivity is very sensitive to cast defects such as cracks in microstructure. It is shown from the Hall measurement that $\mathrm{Al}$ doping increases the carrier concentration [Fig. 3(b)]. The increased carrier concentration in a semimetal such as CoSi would simultaneously enhance the scattering between carriers, leading to an apparent increase in electrical resisitivity by $\mathrm{Al}$ doping.

The temperature dependence of the Seebeck coefficient of $\mathrm{CoSi}_{1-x} \mathrm{Al}_{x}$ single crystals is shown in Fig. 4(a), compared with the data for binary $\mathrm{CoSi}$ (dashed line) reported in the literature. ${ }^{1}$ For comparison, the dependence of the Seebeck coefficient at room temperature on $\mathrm{Al}$ concentration $x$ is plotted in Fig. 4(b) together with the data from the literatures. ${ }^{1,8}$ For binary $\mathrm{CoSi}$, the measured $S$ value is very close to that in the literature ${ }^{1,8}$ (Fig. 4). The negative $S$ values of binary CoSi indicate electron-type conduction over the investigated temperature range, consistent with the previous results. ${ }^{1,2,4}$ Upon $\mathrm{Al}$ substitution, the $S$ value of $\mathrm{CoSi}_{1-x} \mathrm{Al}_{x}$ increases. At room

TABLE I. Compositions of the phases marked as "A" and "B" in Fig. 2 determined by EPMA (in at. \%).

\begin{tabular}{cccc}
\hline \hline Phase & Co & Si & Al \\
\hline A & 51.40 & 37.18 & 11.42 \\
B & 49.98 & 20.36 & 29.66 \\
\hline \hline
\end{tabular}



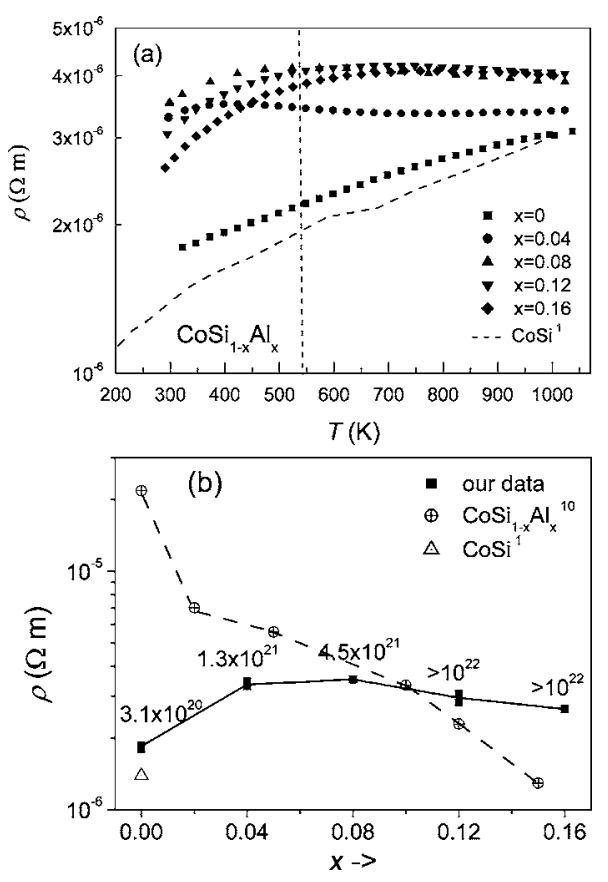

FIG. 3. Electrical resistivity of $\mathrm{CoSi}_{1-x} \mathrm{Al}_{x}$ single crystals (a) vs temperature and (b) at room temperature [the numbers indicate the measured carrier density $\left.\left(\mathrm{cm}^{-3}\right)\right]$.

temperature, the sign of $S$ changes from negative to positive at around $x=0.04$ [Fig. 4(b)]. Contrary to electrical resistivity, our measured $S$ values are very close to the reported data, ${ }^{1,2,4}$ probably due to the reason that the Seebeck coefficient is relatively insensitive to defects in the specimen. Figure 4(a) shows that for the Al-doped specimens, the $S$ value decreases with increasing temperature. The sign of $S$ changes from positive at low temperatures to negative at high temperatures. The temperature at which the sign reverses increases when $\mathrm{Al}$ concentration increases (Fig. 4(a)). For example, when $x=0.04$, the transition temperature is about $320 \mathrm{~K}$, and when $x=0.16$, the transition temperature is about $720 \mathrm{~K}$. This should be attributed to the change of hole concentration by Al doping. The observed Seebeck coefficients can be understood within the framework of a two-carrier conduction model. According to this model, the total $S$ can be expressed as ${ }^{10}$

$$
S=\frac{\sigma_{h} s_{h}+\sigma_{e} s_{e}}{\sigma_{h}+\sigma_{e}},
$$

where $S_{p, n}$ and $\sigma_{p, n}$ represent the Seebeck coefficients and electrical conductivities for the $p$ - and $n$-type carriers, respectively. Since the signs of $S_{p}$ and $S_{n}$ are opposite, increasing the hole concentration by $\mathrm{Al}$ doping could result in a sign change in $S$. This is actually what we observed in the present study. However, the absolute $S$ value of $\mathrm{CoSi}_{1-x} \mathrm{Al}_{x}$ is considerably smaller than that of binary CoSi.

Within the two-carrier conduction scenario, the electron mobility is much higher than that of holes due to the smaller effective mass. Moreover, from the band-structure calculations ${ }^{1,7}$ the electron pockets are larger than the hole pockets. Therefore, the observed Seebeck coefficient of binary $\mathrm{CoSi}$ at room temperature is negative. When $\mathrm{Al}$ substitutes for $\mathrm{Si}$, hole concentration increases leading to a $p$-type
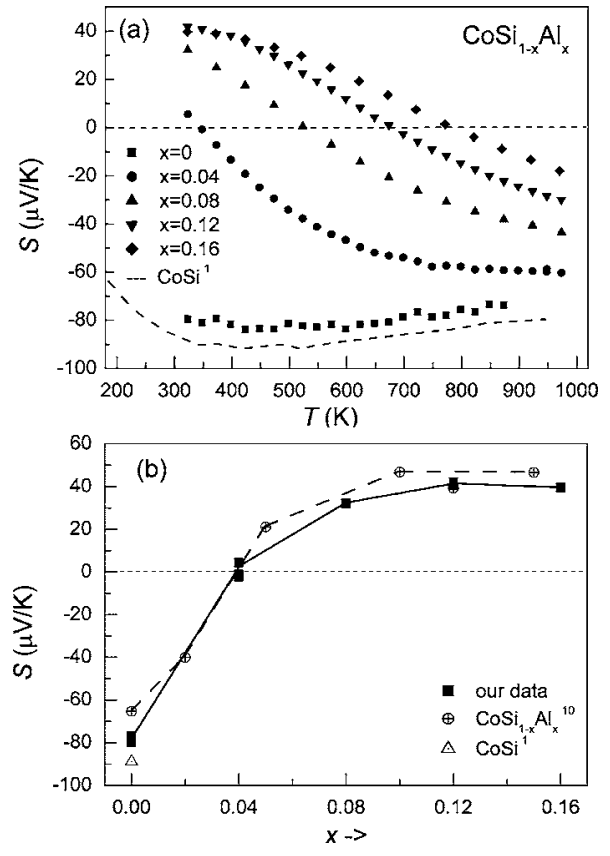

FIG. 4. Seebeck coefficient of $\mathrm{CoSi}_{1-x} \mathrm{Al}_{x}$ single crystals (a) vs temperature and (b) at room temperature.

conductivity, as illustrated in the present study. Our recent band-structure calculation ${ }^{7}$ shows that the Fermi level shifts downwards after $\mathrm{Al}$ doping. This downward shift reduces the bottom of the upper band for the electron but enlarges the top of the lower band for the hole, yielding the $p$-type carriers that dominate the transport behavior. This is highly consistent with the present observations. With increasing $\mathrm{Al}$ concentration, it is reasonable to anticipate that the hole concentration increases further. Therefore, more thermally excited electrons are needed to balance the extra holes. This may explain why $S$ changes its sign at a higher temperature under a higher $\mathrm{Al}$ concentration.

Figure 5(a) shows the temperature dependence of the thermal conductivity of $\mathrm{CoSi}_{1-x} \mathrm{Al}_{x}$ single crystals. Compared to the data for binary $\mathrm{CoSi}$ polycrystal, ${ }^{1}$ the measured thermal conductivity of CoSi single crystal is slightly lower. The thermal conductivity of the binary CoSi crystal decreases with temperature. The effect of Al substitution on lowering the thermal conductivity is clear from the plot. For Al-doped specimens, a general trend in thermal conductivity is that the thermal conductivity first decreases and then increases slightly with temperature. For the heavily doped specimen $(x=0.16)$, the thermal conductivity decreases continuously with temperature, probably due to the reason that the transition temperature is outside the investigated temperature range.

Thermal conductivity $\kappa$ for semimetals can be divided into two parts: the carrier contribution $\kappa_{\text {car }}$ and the lattice contribution $\kappa_{\text {lat }}$. The carrier contribution part $\kappa_{\text {car }}$ can be estimated from the Wiedemann-Franz relationship $\kappa_{\text {car }}$ $=L_{0} T / \rho$, where $L_{0}$ is the Lorentz number $\left(L_{0}=2.44\right.$ $\left.\times 10^{-8} \mathrm{~W} \Omega / \mathrm{K}^{2}\right),{ }^{10} \rho$ is the electrical resistivity, and $T$ is the absolute temperature. The lattice contribution $\kappa_{\text {lat }}$ is obtained by subtracting $\kappa_{\text {car }}$ from the observed thermal conductivity $\kappa$. As shown in Fig. 5(b), the lattice contribution $\kappa_{\text {lat }}$ is pre- 

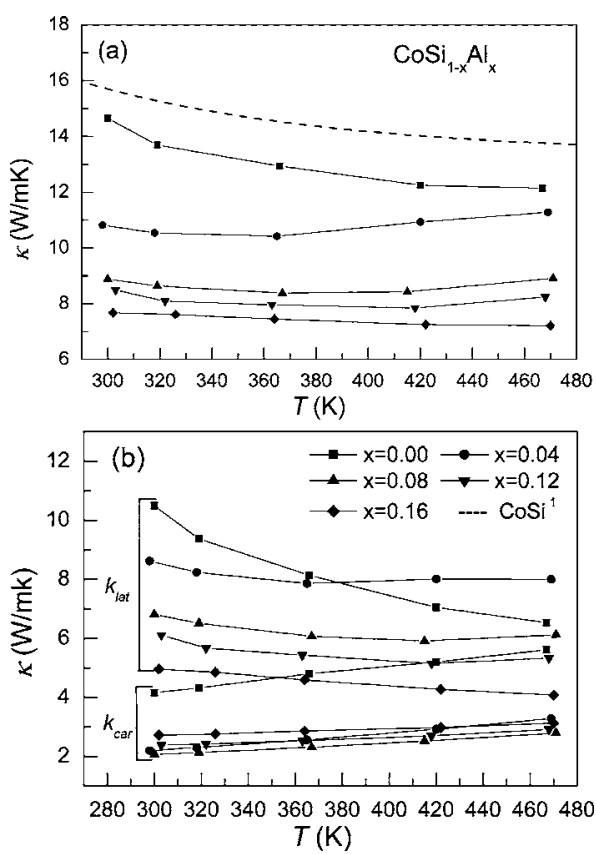

FIG. 5. (a) Thermal conductivity of $\mathrm{CoSi}_{1-x} \mathrm{Al}_{x}$ single crystals vs temperature. (b) Lattice thermal conductivity $\left(k_{\text {lat }}\right)$ and carrier thermal conductivity $\left(k_{\text {car }}\right)$ of $\mathrm{CoSi}_{1-x} \mathrm{Al}_{x}$ single crystals vs temperature.

dominant in the total thermal conductivity. The electron contribution of all the investigated specimens increases almost linearly with temperature. This may be attributed to an increase in thermally activated carrier density. The temperature dependence of the lattice contribution of binary $\mathrm{CoSi}$ is roughly proportional to $1 / T$, indicating that the decrease in thermal conductivity is mainly caused by thermal vibration. Both the lattice and electron contributions decrease after $\mathrm{Al}$ doping. For the doped specimens, the temperature dependence of the lattice contribution is relatively weak, which is different from that of the binary one. This may imply that $\mathrm{Al}$ substitution for $\mathrm{Si}$ in $\mathrm{CoSi}$ would introduce additional phonon scattering which in turn results in a decreased thermal conductivity. Since the additional scattering is caused by the dopant $\mathrm{Al}$ instead of the thermally activated carrier, the temperature dependence of thermal conductivity would be weak. Lue et al. ${ }^{10}$ has also observed a similar decrease in thermal conductivity by $\mathrm{Al}$ doping and attributed it to point defects.

In practical application, the performance of a thermoelectric material is characterized by the dimensionless figure of merit $Z T=S^{2} T / \rho \kappa .{ }^{11}$ Figure 6 shows $Z T$ value as a function of temperature for all the samples. It can be found that Al doping significantly decreases the $Z T$ of $\mathrm{CoSi}$. This is largely attributed to the significant decrease of electrical transport properties by $\mathrm{Al}$ doping, though thermal conductivity decreases after doping in the meanwhile.

\section{CONCLUSION}

The effects of $\mathrm{Al}$ substitution for $\mathrm{Si}$ on the thermoelectric performance of $\mathrm{CoSi}$ were investigated in the temperature range from 300 to $973 \mathrm{~K}$. The solubility limit of $\mathrm{Al}$ in

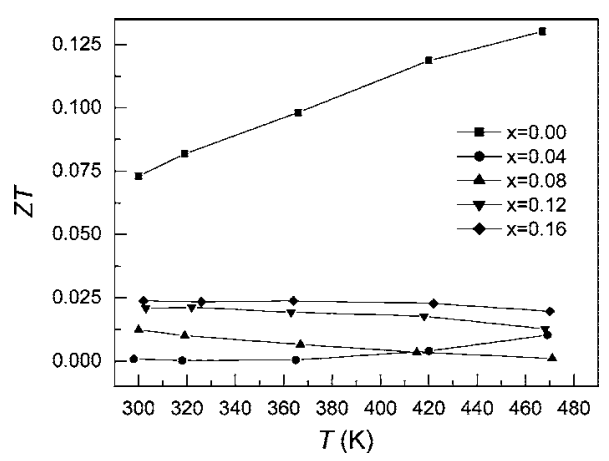

FIG. 6. $Z T$ values of $\operatorname{CoSi}_{1-x} \mathrm{Al}_{x}$ single crystals vs temperature.

$\mathrm{CoSi}_{1-x} \mathrm{Al}_{x}$ is found to be in the range of $x=0.20-0.30$. The $B 2$-type CoAl phase forms in the microstructure when $x$ $\geqslant 0.3$. There is substantial increase in electrical resistivity upon Al substitution. At high doping levels $(x \geqslant 0.08)$, the electrical resistivity decreases slightly in the temperature range from 300 to $550 \mathrm{~K}$ and remains almost unchanged at higher temperatures with increasing $x$. The Seebeck coefficient of the undoped $\mathrm{CoSi}$ is negative within the investigated temperature range. For the substituted specimens, the sign of the Seebeck coefficient changes from positive to negative with increasing temperature. The reverse point is at a higher temperature with increasing $\mathrm{Al}$ concentration. However, the absolute value of the Seebeck coefficient is lowered by Al substitution. Thermal conductivity is significantly reduced by $\mathrm{Al}$ substitution. Both the lattice and carrier contributions to thermal conductivity are decreased, but the decrease in lattice contribution is much larger. The $Z T$ of CoSi is significantly reduced by $\mathrm{Al}$ substitution. This mainly results from the decrease in electrical transport properties.

\section{ACKNOWLEDGMENTS}

This work was supported by the Shanghai-Applied Materials Research and Development Foundation (Contract No. 0317) and the National Natural Science Foundation of China (Contract No. 50131030). One of the authors (W.L.R.) acknowledges the China and Shanghai Postdoctoral Science Foundation for their financial support.

${ }^{1}$ D. M. Rowe, CRC Handbook of Thermoelectrics (CRC, Boca Raton, FL, 1995).

${ }^{2}$ S. Asanabe, D. Shinoda, and Y. Saski, Phys. Rev. 134, A774 (1964).

${ }^{3}$ G. T. Alekseeve, V. K. Zailsev, A. V. Petrov, V. I. Tarasov, and M. I. Fedorov, Sov. Phys. Solid State 23, 1685 (1981).

${ }^{4}$ S. W. Kim, Y. Mishima, and D. C. Choi, Intermetallics 10, 177 (2002).

${ }^{5}$ E. N. Nikitin, P. V. Tamarin, and V. I. Tarasov, Sov. Phys. Solid State 11, 2002 (1970).

${ }^{6}$ Y. Imai, M. Mukaida, K. Kobayashi, and T. Tsunoda, Intermetallics 9, 261 (2001).

${ }^{7}$ Z. J. Pan, L. T. Zhang, and J. S. Wu, Acta Phys. Sin. 54, 328 (2005).

${ }^{8}$ W. L. Ren, C. C. Li, L. T. Zhang, K. Ito, and J. S. Wu, J. Alloys Compd. 392, 50 (2005).

${ }^{9}$ Y. Zhou, C. C. Li, W. L. Ren, L. T. Zhang, and J. S. Wu, Chin. J. Nonferrous Met. 14, 2055 (2004).

${ }^{10}$ C. S. Lue, Y. K. Kuo, C. L. Huang, and W. J. Lai, Phys. Rev. B 69, 125111 (2004).

${ }^{11}$ G. D. Mahan, J. Appl. Phys. 65, 1578 (1989). 